\title{
Favipiravir, will it be the Answer for the Specific Management of COVID-19?: A Review
}

\author{
Jayant Patharkar'1, Maulin Mehta' ${ }^{2}$ Janhavi Patharkar $^{3}$ \\ ${ }^{1}$ Assistant Professor, ${ }^{2}$ Associate Professor,Department of Pharmacology, Smt. B.K. Shah Medical Institute \& \\ Research Centre, Sumandeep Vidyapeeth Deemed to be University, At. \& Post. PIPARIA, Tal. Waghodia, Dist. \\ Vadodara-391760, Gujarat State, ${ }^{3}$ Assistant Professor, Department of Kayachikitsa, Parul Institute of Ayurved, At. \\ \& Post. Limda, Tal. Waghodia, Dist. Vadodara-391760, Gujarat state.
}

\begin{abstract}
Background: The COVID-19 has created havoc all over the world as no specific treatment available till date. Total COVID-19 cases had crossed 11,125,245 including 528,204 deaths as per the WHO data till 5 July 2020. Unfortunately, India secured third place in total tally of COVID-19 cases with $6,77,719$ total cases including 19693 deaths as of $5^{\text {th }}$ July 2020. The antiviral drug, Favipiravir, previously known as T-705 approved in Japan for treatment of Influenza. It is selective \& potent inhibitor of viral RNA polymerase. It is promising drug for treatment of wide range of RNA viruses including SARSCoV-2.

Method: The systematic review of Articles was done with the help of search engines like Embase, Medline, PubMed \& Google Scholar, clinicaltrials.gov. The Peer reviewed articles published through $6^{\text {th }}$ of July 2020 were included in the study. This review was done to establish clinical evidence for use of Favipiravir in COVID-19.
\end{abstract}

Conclusion: It was found that due to lack of conclusive evidence, Favipiravir cannot be included in main armamentarium for COVID-19.

Keywords: COVID-19 management, clinical trials review, Favipiravir, repurpose drug.

\section{Introduction}

The COVID-19 has created havoc all over the world as no specific treatment available till date. Total COVID-19 cases had crossed 11,125,245 including 528,204 deaths as per the WHO data till 5 July $2020^{[1]}$. Unfortunately, India secured third place in total tally of COVID-19 cases with $6,77,719$ total cases including 19693 deaths as of $5^{\text {th }}$ July $2020^{[2]}$.

\section{Corresponding Author:}

\section{Dr Jayant T Patharkar}

Assistant Professor, Department of Pharmacology, Smt. B.K. Shah Medical Institute \& Research Centre, Sumandeep Vidyapeeth Deemed to be University, At.

\& Post. Piparia, Tal. Waghodia, Dist. Vadodara-391760, Gujarat State

e-mail: jayant.patharkar@gmail.com

Mob No.: 7046519567
The antiviral drug, Favipiravir, previously known as T-705 approved in Japan for treatment of Influenza. It is prodrug of a purine nucleotide i.e. Favipiravir ribofuranosyl-5'-triphosphate. It is selective $\&$ potent inhibitor of viral RNA polymerase. Thus, it halts the viral replication. It is effective against all strains of influenza viruses. It is promising drug for treatment of wide range of RNA viruses including SARSCoV $-2^{[3,4]}$.

At present, there is no robust evidence from randomised clinical trials (RCTs), that specific drug or treatment will improve the patient outcome of COVID-19. Thus, various treatment modalities, repurposed or experimental drugs are under the clinical trials for establishing evidence based specific treatment for novel corona virus epidemic. The repurposed drugs under such clinical trials are Chloroquine phosphate, hydroxychloroquine sulfate, Lopinavir/ritonavir, Umifenovir (Arbidol), Remdesivir, Favipiravir, Tocilizumab $^{[5]}$. 
Hence, this review was proposed for Flavipiravir, to understand current $\&$ future prospect of this promising antiviral drug by summarizing various clinical trials, studies \& analysing the outcome of these studies.

\section{Method}

The systematic review of Articles was done with the help of search engines like Embase, Medline, PubMed \& Google Scholar, clinicaltrials.gov. The Peer reviewed articles published through $6^{\text {th }}$ of July 2020 were included in the study. The Search words used in combinations included; COVID-19, Favipiravir, Antiviral, COVID-19 specific treatment options, repurposed drugs, Randomized Clinical Trials (RCTs)

All the studies, randomized clinical trials, cohort, prospective, metanalysis, review was included in for the review, keeping the goal as to ascertain the role of Favipiravir in the management of COVID-19.

\section{Results}

Various studies conducted for COVID-19 patients treated with Favipiravir:

1. The Clinical Trials gov Identifier: NCT04349241 by Hany Dabbous, Ain Shams University in 2020, sample size was $100 \&$ intervention was Favipiravir. The out come of this trial is not available

2. A Randomized Clinical Trial: Favipiravir versus Arbidol for COVID conducted by Chang Chen, Ping Yin, Yi Zhang, Jianying Huang et al. in 240, sample size was 240 \& intervention was Favipiravir, Arbidol. In this trial they found that Favipiravir significantly improved the latency to relief for fever and cough.

3. An Open-Label Control Study for COVID: Experimental Treatment with Favipiravir by Cai a Qingxian,y, Minghui Yang a,y, Dongjing Liu a,y, Jun Chen a,y, Dan Shu a, Junxia Xia a, Xuejiao in the year 2020. The sample size was 80 with intervention used Favipiravir (FPV) versus Lopinavir (LPV)/ ritonavir (RTV). In this study significant difference observed $(p=0.004)$ in radiological parameters like chest imaging when favipiravir used against control group.

4. The ClinicalTrials.gov Identifier: NCT01068912 conducted by MDVI, LLC in year 2015, in sample of 530, wherein they used Favipiravir against placebo comparator. In this trial they found that the Adverse drug reactions, deaths were not seen in influenza patients when favipiravir used $\&$ it was proved to be effective for different doses.

5. The ClinicalTrials.gov Identifier: NCT02026349 by MDVI, LLC in 2015 among 860 patients found that the patients of influenza were cured of symptoms like temperature without any untoward side effects with favipiravir.

6. The Clinical trial, Identifier: NCT04376814 conducted by Baqiyatallah Medical Sciences University among 40 patients, wherein favipiravir compared with another arm having Hydroxychloroquine, Lopinavir/Ritonavir. The result of this trail was not reported.

7. The Bioequivalence Study of Favir $200 \mathrm{mg}$ Film Tablet Kocak Under Fasting conditions, Clinical trials gov Identifier NCT04444986 by Kocakfarma in 2020 among 30 patients using Favir 200 mg FT against Avigan $200 \mathrm{mg}$ FT. The result of study was not reported.

8. A Review: Pharmacologic Treatments for Coronavirus Disease 2019 was conducted by James M. Sanders, L. Monogue, Marguerite et al. in 2020 found that no therapies have been shown effective to date.

9. A systematic review and meta-analysis: Efficacy and safety of antiviral treatment for COVID-19 from evidence in studies of SARSCoV-2 and other acute viral infections conducted by Wei Liu, Pengxiang Zhou, Ken Chen, Zhikang Ye, Fang Liu, et al. in 2020 found that presently no existing antiviral treatment beneficial.

10. A Review Pharmacological Therapeutics Targeting RNA-Dependent RNA Polymerase, Proteinase and Spike Protein: From Mechanistic Studies to Clinical Trials by JianshengHuang, Hui Huang Wenliang Song in 2020 commented that with the development of potent \& effective RdRp inhibitors will be useful in SARS-CoV-2 therapy.

\section{Discussion}

The Favipiravir, repurposed drug for COVID-19 treatment. It has been the approved drug for use in the influenza in Japan. Favipiravir is a prodrug which is converted intracellularly into phosphoribosylated form-active form. This active form is recognized as a substrate by the viral RNA-dependent RNA polymerase. $\&$ replication of virus can be prevented ${ }^{[6]}$. 
In the Clinical trials registries, total 21 studies have been registered for use of Favipiravir in COVID-19 patients. Out of these studies three studies are completed $\&$ only two studies result has been published. Out of these two studies, the multi-centric phase II trial (NCT01068912), has shown clinical efficacy of Flavipiravir in Influenza. This study also posted that the clinical efficacy can be seen in low-dose as well as high-dose regimens, no serious adverse events has been witnessed ${ }^{[7]}$. In another study, which posted result (NCT02026349), it was noted that favipiravir could reduce symptoms and fever could be subsided without any adverse events ${ }^{[8]}$.

In the randomized controlled trial conducted by Chang Chen et al.in March 2020, found that the patient had relieved of pyrexia \& cough in short span of time with Favipiravir significantly, but no significant change has been seen in clinical recovery rate as compared to Arbidol.

An Open-Label Control Study conducted with favipiravir \& control arm with Lopinavir/ritonavir in COVID-19 patients elucidated that radiological improvement seen when viral load was reduced early. It is noteworthy that less adverse events occurred with Favipiravir than control arm having Lopinavir (LPV)/ ritonavir (RTV). In this trial treatment Favipiravir given orally (200 mg per tablet), dose $1600 \mathrm{mg}$ two times in a day on first day \& two times in a day for next thirteen days. The Lopinavir (200 mg)/Ritonavir (50 mg) were taken through enteral route, two tablets each two times in a day. On first day \& then maintained for next thirteen days two times in a day ${ }^{[9]}$. Thus, in this study, efficacy in reducing viral load as well as safety of favipiravir over Lopinavir/ritonavir can be ascertained in the patients of COVID-19.

A treatment review by James M. Sanders et al. of Coronavirus disease found that, the preclinical data obtained about favipiravir was from its activity against influenza \& Ebola virus. In Vero E6 cells, in vitro studies, the EC50 of favipiravir against SARSCoV-2 was $61.88 \mu \mathrm{M} / \mathrm{L}^{[10]}$. The doses would differ according to type of infection. Thus, for COVID-19, higher dosing range was selected i.e. a maintenance dose $(1200 \mathrm{mg}$ to $1800 \mathrm{mg}$ every 12 hours) after loading dose (2400 mg to $3000 \mathrm{mg}$ every 12 hours $\times 2$ doses $)^{[11,12]}$. Favipiravir is being used in influenza in Japan but it is not available in the united states. Thus, to establish the evidence for use of favipiravir in the SARSCo-2, more RCTs has to be conducted in near future ${ }^{[5]}$.

A systematic review \& meta-analysis about the efficacy \& safety of antiviral drugs in COVID-19, stated that there is no evidence present currently to confirm important benefits of antiviral treatment but still did not strike out important benefits of individual antiviral agent $^{[13]}$.

Jiansheng Huang revived about target proteins like RNA-Dependent RNA Polymerase, spike proteins \& proteinase in various clinical trials and studies being conducted during this COVID-19 pandemic and on the basis of mechanism of replication of SARS-CoV-2 RNA-Dependent RNA Polymerase, found that effective \& potent RdRp inhibitors may play key role in the treatment of COVID-19 ${ }^{[14]}$. At present Favipiravir, inhibitor of RNA polymerase is used for influenza in Japan. However, the safety issue about favipiravir should be kept in mind because of its teratogenic risk though this broad-spectrum antiviral seems promising.

\section{Conclusion}

The Favipiravir, repurpose drug, had shown promising results in COVID-19 in few of the studies but vast majority of clinical trials are ongoing. Hence at this juncture, it will be overambitious to put Favipiravir as front warrior against COVID-19. Thus, due to lack of conclusive evidence, Favipiravir cannot be included in main armamentarium for COVID-19.

\section{Research funding: None}

\section{Financial support and sponsorship: Nil}

\section{Conflict of Interest: None}

Ethical Clearance:It was obtained from Sumandeep Vidyapeeth Institutional Ethics Committee before starting the study.

\section{References}

1. The current cases of COVID-19 in the world, https:// covid19.who.int/(Accessed on $6^{\text {th }}$ July 2020)

2. The current cases of COVID-19 in India, https:// www.mygov.in/covid-19 (Accessed on $6^{\text {th }}$ July 2020)

3. Furuta Y, Takahashi K, Kuno-Maekawa M, Sangawa H, Uehara S, Kozaki K, Nomura N, Egawa H, Shiraki K. (2005) Mechanism of action of T-705 against influenza virus. Antimicrobial agents and chemotherapy.;49(3):981-986. 
4. Furuta Y, Gowen BB, Takahashi K, Shiraki K, Smee DF, Barnard DL.(2013) Favipiravir (T-705), a novel viral RNA polymerase inhibitor. Antiviral research. 2013 Nov 1;100(2):446-54.

5. Sanders JM, Monogue ML, Jodlowski TZ, Cutrell JB. Pharmacologic treatments for coronavirus disease 2019 (COVID-19): a review. Jama. 2020 May 12;323(18):1824-36.

6. Delang L, Abdelnabi R, Neyts J. Favipiravir as a potential countermeasure against neglected and emerging RNA viruses. Antiviral research. 2018 May 1; 153:85-94.

7. https:// c linicaltrials.gov/ct2/ results?cond=COVID-19 \& term=Favipiravir (Accessed on $7^{\text {th }}$ july 2020)

8. Khambholja K, Asudani D. Potential repurposing of Favipiravir in COVID-19 outbreak based on current evidence. Travel Medicine and Infectious Disease. 2020 Apr 28, 35: 101710.

9. Cai Q, Yang M, Liu D, Chen J, Shu D, Xia J, Liao X, Gu Y, Cai Q, Yang Y, Shen C. Experimental treatment with favipiravir for COVID-19: an openlabel control study. Engineering. 2020 Mar 18.

10. Wang M, Cao R, Zhang L. Remdesivir and chloroquine effectively inhibit the recently emerged novel coronavirus (2019 nCoV) in vitro [published online February 4, 2020]. Cell Res,30;pages269-271

11. Mentré F, Taburet AM, Guedj J, Anglaret X, Keïta S, de Lamballerie X, Malvy D. Dose regimen of favipiravir for Ebola virus disease. The Lancet Infectious Diseases. 2015 Feb 1;15(2):150-1.

12. Sissoko D, Laouenan C, Folkesson E, M'lebing AB, Beavogui AH, Baize S, Camara AM, Maes P, Shepherd S, Danel C, Carazo S. Experimental treatment with favipiravir for Ebola virus disease (the JIKI Trial): a historically controlled, single-arm proof-of-concept trial in Guinea. PLoS medicine. 2016 Mar 1;13(3):e1001967.

13. Liu W, Zhou P, Chen K, Ye Z, Liu F, Li X, He N, Wu Z, Zhang Q, Gong X, Tang Q. Efficacy and safety of antiviral treatment for COVID-19 from evidence in studies of SARSCoV-2 and other acute viral infections: a systematic review and metaanalysis. CMAJ.July 06, 2020192 (27) E734-E744

14. Huang J, Song W, Huang H, Sun Q. Pharmacological therapeutics targeting RNA-dependent RNA polymerase, proteinase and spike protein: from mechanistic studies to clinical trials for COVID-19. Journal of Clinical Medicine. 2020 Apr;9(4):1131. 